\title{
GCU
}

Glasgow Caledonian

University

University for the Common Good

\section{Is there a preference for PET or SPECT brain imaging in diagnosing dementia? The views of people with dementia, carers, and healthy controls}

Bamford, Claire; Olsen, Kirsty; Davison, Chris; Barnett, Nicky ; Lloyd, Jim; Williams, David; Firbank, Michael; Mason, Helen; Donaldson, Cam; O'Brien, John

Published in:

International Psychogeriatrics

DOI:

$10.1017 / \mathrm{S} 1041610215001039$

Publication date:

2016

Document Version

Author accepted manuscript

Link to publication in ResearchOnline

Citation for published version (Harvard):

Bamford, C, Olsen, K, Davison, C, Barnett, N, Lloyd, J, Williams, D, Firbank, M, Mason, H, Donaldson, C \&

O'Brien, J 2016, 'Is there a preference for PET or SPECT brain imaging in diagnosing dementia? The views of people with dementia, carers, and healthy controls', International Psychogeriatrics, vol. 28, no. 1, pp. 123-131. https://doi.org/10.1017/S1041610215001039

\section{General rights}

Copyright and moral rights for the publications made accessible in the public portal are retained by the authors and/or other copyright owners and it is a condition of accessing publications that users recognise and abide by the legal requirements associated with these rights.

Take down policy

If you believe that this document breaches copyright please view our takedown policy at https://edshare.gcu.ac.uk/id/eprint/5179 for details of how to contact us. 
Is there a preference for PET or SPECT brain imaging in diagnosing dementia? The views of people with dementia, carers and healthy controls

Authors

Claire Bamford ${ }^{1}$

Kirsty Olsen²

Chris Davison ${ }^{2}$

Nicky Barnett ${ }^{2}$

$\operatorname{Jim}$ Lloyd $^{3}$

David Williams ${ }^{2}$

Michael Firbank ${ }^{2}$

Helen Mason ${ }^{4}$

Cam Donaldson ${ }^{4}$

John O'Brien ${ }^{2,5}$

1 Institute of Health and Society, Newcastle University, Newcastle upon Tyne, NE4 5PL, UK

2 Institute of Neuroscience, Campus for Ageing and Vitality, Newcastle University, Newcastle upon Tyne, NE4 5PL, UK

3 Regional Medical Physics Department, Newcastle upon Tyne Hospitals NHS Foundation Trust, UK

4 Yunus Centre for Social Business and Health, Glasgow Caledonian University, Glasgow, G4 OBA, UK Department of Psychiatry, University of Cambridge School of Clinical Medicine, Box 189, Cambridge Biomedical Campus, Cambridge, CB2 0SP, UK 


\section{Corresponding author:}

Claire Bamford

Institute of Health and Society, Newcastle University, Newcastle Biomedical Research Building, Campus for Ageing and Vitality, Newcastle upon Tyne, NE4 5PL, UK

Email: claire.bamford@ncl.ac.uk

Tel: $\quad+44(0) 1912087047$

Fax: $\quad+44(0) 1912086043$ 


\section{Abstract}

Background: PET and SPECT brain imaging are widely used as diagnostic tools for suspected dementia but no studies have directly compared participant views of the two procedures. We used a range of methods to explore preferences for PET and SPECT. Methods: Patients and controls (and accompanying carers) completed questionnaires immediately after undergoing PET and SPECT brain scans. Pulse rate data were collected during each scan. Scan attributes were prioritised using a card sorting exercise; carers and controls additionally answered willingness to pay questions.

Results: Few differences were found either between the scans or groups of participants, although carers marginally preferred SPECT. Diagnostic accuracy was prioritised over other scan characteristics. Mean heart rate during both scans was lower than baseline heart rate measured at home $(p<.001)$.

Conclusion: Most participants viewed PET and SPECT scans as roughly equivalent and did not have a preference for either scan. Carer preference for SPECT is likely to reflect their desire to be with the patient (routine practice for SPECT but not for PET), suggesting that they should be able to accompany vulnerable patients throughout imaging procedures wherever possible. Pulse rate data indicated that brain imaging was no more stressful than a home visit from a researcher. The data do not support the anecdotal view that PET is a more burdensome procedure and the use of PET or SPECT scans in dementia should be based on diagnostic accuracy of the technique.

Key words: PET, SPECT, Diagnosis, Alzheimer's disease, dementia with Lewy bodies, Patient preference; willingness to pay

Running title: Preferences for PET or SPECT brain imaging 


\section{Introduction}

Distinguishing dementia subtypes facilitates optimal management and enables the provision of appropriate information to patients and carers about likely symptomatology and prognosis. Clinical trials also rely on accurate diagnosis of dementia subtypes. Current guidelines recommend blood flow (hexamethylpropyleneamine oxime, HMPAO) Single Photon Emission Computed Tomography (SPECT) or functional brain imaging using glucose (fludeoxyglucose, FDG) Positron Emission Tomography (PET) to facilitate differential diagnosis of the cause of the dementia syndrome when diagnosis is in doubt (Gauthier, 2012; Hort et al., 2010; NICE/SCIE, 2006). There is growing evidence that PET is more accurate as a diagnostic tool (Herholz, 2011; Ishii and Minoshima, 2005; O'Brien et al., 2014). Decisions about the use of PET or SPECT may also be influenced by availability, financial cost and perceived acceptability to patients. SPECT has been more readily available and significantly cheaper than PET (Colloby and O'Brien, 2004). However, PET is now more widely available and the price of FDG, used for PET, has reduced significantly over the last ten years (O'Brien et al., 2014). Anecdotally PET is perceived as more burdensome for patients than SPECT (NICE/SCIE, 2006) but little is known about the patient experience of undergoing imaging or patient preferences (Davison and O'Brien, 2014). Although PET and SPECT are both nuclear medicine scans, the procedures are more involved for PET than for SPECT. For example, patients have to fast and have their glucose checked prior to PET and while carers are able to stay with patients throughout SPECT scans, they are usually not allowed in the room during PET scans due to concerns about their exposure to the higher level of radiation associated with PET. Patient views on the acceptability of SPECT and PET could help inform future decisions regarding the most appropriate imaging procedure (Davison and O'Brien, 2013).

As part of a study which investigated the diagnostic utility of PET with SPECT in distinguishing between people with neurodegenerative dementia (Alzheimer's disease (AD) and DLB) and normal controls (O'Brien et al., 2014), we collected data on participants' views 
of SPECT and PET brain imaging. Our aim was to develop a detailed understanding of participant views and experiences of the two scans and to explore whether the anecdotal view that PET is more burdensome and less patient friendly than SPECT was supported by empirical data.

\section{Methods}

\section{Study population}

Patients aged over 60 with mild to moderate dementia (MMSE>12) (Folstein et al., 1975) who met the criteria for probable AD (McKhann et al., 1984) or probable DLB (McKeith et al., 2005) were recruited prospectively from clinical services in north east England. We also recruited age-matched controls with no signs or symptoms of dementia who had expressed a willingness to participate in research studies. All participants underwent detailed neuropsychiatric investigation prior to imaging (O'Brien et al., 2014). SPECT and PET scans were completed on separate occasions within one month. The order of the scans was alternated between consecutive subjects in each group. The views of carers accompanying patients to the scans were also sought.

SPECT head scans were undertaken using a Siemens Symbia dual-detector gamma camera. Subjects were comfortably seated in a quiet room, with eyes open for a bolus intravenous injection of $500 \mathrm{MBq}$ of Tc-99m HMPAO (Ceretec (exametazime)). They then either returned to the nuclear medicine waiting room for up to 30 minutes or proceeded directly to the camera room. Imaging time was 25 minutes. This process reflected the standard practice in a busy clinical department.

PET-CT scans were undertaken using a Siemens Biograph Truepoint PET-CT. Imaging took place approximately 30 minutes after intravenous injection of $250 \mathrm{MBq}{ }^{18} \mathrm{~F}-\mathrm{FDG}$ (fluorodeoxyglucose). Subjects were asked to fast for four hours pre-injection and blood glucose was tested. During the injection, subjects were comfortably seated in a quiet room, 
with eyes open. They then remained alone in an individual cubicle until their scan. Imaging took approximately ten minutes. Although the equipment and procedures used were similar to those used in clinical settings, the PET scans took place in a university clinical research facility, while the SPECT scans were done in a UK NHS hospital department. All scans were performed by NHS staff.

\section{Data collection}

An overview of all data collection procedures is provided in Table 1. The development, content and administration of each measure are described below.

[Insert Table 1 about here please]

\section{Questionnaires}

While patients have made direct comparisons of different diagnostic procedures in previous studies (Liang et al., 2003; Sparrow et al., 2004), people with dementia were unlikely to be able to recall details of the imaging procedures after any length of time. To capture participants' experiences and views on the two scans, the questionnaires were therefore administered immediately after each scan.

Parallel questionnaires were produced for patients, controls and carers accompanying patients to the scan. The content of the questionnaires was informed by:

- existing literature on patient views of diagnostic procedures (Sparrow et al., 2004; Wollman et al., 2004);

- observation of routine practice in PET and SPECT suites; and

- $\quad$ semi-structured interviews with five people with dementia referred for SPECT imaging and accompanying carers (these participants were not part of the main study). 
Questionnaires for patients and controls were interviewer-administered and took around ten minutes to complete. The carer questionnaire was usually self-completed. Three open questions were included on each questionnaire to elicit views on the best and worst things about the scan and to identify any suggested improvements. Three further questions were added after use with the first five participants and carers recruited to the main study.

\section{Prioritising task}

We used a simplified version of $Q$ methodology (Baker et al., 2006) to explore the relative priority given to attributes of the scan identified as potentially important in the development work:

- Being able to get comfortable in the scanner

- Length of time for the injection and scan

- Dose of radiation given

- Accuracy of the scan

- How far you have to travel for the scan

- How confined you feel in the scanner

- Whether my relative can stay in the room

- Helpful staff

- Noise of the scanner

A further item - having to fast before the scan - was added after the first five participants and carers recruited to the main study. Each item was printed on a card and respondents were asked to sort the cards according to importance. Additional blank cards were available so that respondents could add other factors if needed. Since the prioritising task did not require detailed recall of the scans it was completed during a final home visit after completion of both scans. 


\section{Willingness to pay questionnaire}

The willingness to pay (WTP) questions were completed during the final home visit. We used an 'incremental approach' (Donaldson and Shackley, 1997) whereby participants were asked which scan they preferred (PET or SPECT) and the maximum amount they would be willing to pay for their preferred option. We presented cards with amounts ranging from $£ 1$ to $£ 5000$ in random order and asked respondents to sort the cards into three piles: definitely willing to pay that amount; definitely not willing to pay; or unsure. Any cards in the unsure pile were then reviewed to identify the maximum amount the respondent was willing to pay for the preferred scan. Due to concerns over the ability of people with dementia to remember and compare both scans, ethical concerns over potential confusion over whether they would be required to pay for the procedures and desire to minimise respondent burden, the WTP questions were not administered to patients.

\section{Physiological measurement}

Information on average heart rate was collected as an indicator of stress (Sparrow et al., 2004) using a fingertip pulse oximeter (Pulmolink CMS 50E-W). Normal heart rate was ascertained by measurements taken for five minutes during the initial home visit. Further pulse data were collected for five minutes on arrival at the department and throughout each scan

\section{Study approvals}

The study was sponsored by Northumberland, Tyne and Wear NHS Foundation Trust. Ethical approval was given by Newcastle and North Tyneside 1 Research Ethics Committee (reference 09/H090688).

\section{Statistical analysis}

All questionnaire items were coded so that higher scores indicate more positive views. To test for differences in views of PET and SPECT scans, questions were compared within 
respondent type (patients, controls, carers) using the Wilcoxon Signed-Ranks test for questions with scaled response categories and the McNemar test for those with dichotomous responses. Views of patients and controls on each type of scan were compared using a Mann Whitney test for two independent samples. Responses to the open questions were recoded as a series of binary variables and compared across all three groups of respondents (Kruskal Wallis test for independent samples).

The priority given to each attribute of the scan ranked in the prioritising task was recoded as important (ranked most, second most or third most important), unimportant (ranked as least, second least or third least important) or as neither important nor unimportant (all items not ranked at either extreme). The priorities of patients, controls and carers were compared using the Kruskal Wallis test for independent samples. The WTP data were subject to simple descriptive analyses and a Mann Whitney $U$ test to compare the amounts respondents were willing to pay for their preferred scan.

Since the PET scans were considerably shorter than SPECT scans (approximately ten vs 25 minutes) we divided the pulse rate data into eight minute segments to directly compare the two scans. (This allowed some time for the pulse rate to stabilise at the start of the recording). The first eight minute segment was subdivided into two four minute segments to allow us to compare pulse rate during these two segments of both scans; the second and third 8 minute segments of pulse data for the SPECT scan were used to explore whether participants found the scan more or less stressful as duration of scan increased (comparable data for PET not being available). Pulse rate data were analysed using analysis of variance.

\section{Results}

\section{Response rates}

We recruited 102 subjects, of whom 3 withdrew before completing both scans, and 1 was excluded due to scanner technical problems. A total of 38 people with $A D, 30$ with DLB and 
30 controls were successfully scanned with both PET and SPECT. Questionnaire data were not completed by the first eight people recruited to the study ( 5 controls, 3 patients) since the questionnaires were awaiting ethical approval. The questionnaires for one further patient were unavailable. Patients and controls were matched on age (mean age 76, standard deviation (SD) 6.4 years) and gender (69\% male). As expected, patients had significantly lower MMSE scores (mean 21, SD 3.9) than controls (mean 29, SD 1.1, p<0.001, t-test) and were also more likely to have previously undergone a SPECT scan ( $16 \%$ patients vs $0 \%$ of controls, $p=0.03$, Chi square).

For those patients completing questionnaires, the average duration of dementia was 39.8 months (range 9-120 SD 22 months); $53 \%$ had a diagnosis of AD and 47\% DLB. A carer accompanied 62 patients to both scans and 60 of these completed post-scan questionnaires. One carer was unavailable at the final home visit and therefore the analysis of the prioritising task and WTP questionnaire is based on 59 carers.

\section{Preference questionnaires}

Comparison of responses for PET and SPECT indicated minimal differences (Figure 1).

[Insert Figure 1 about here please]

Carers' views were broadly similar to those of patients and controls. For patients and carers the key difference between the scans was, as expected, whether the carer was in the room during the scan (PET: $0 \%$ for patients and carers; SPECT: $70.3 \%$ patients and $69.5 \%$ carers;; $p<0.001$ for both patients and carers, McNemar test). The subjective feeling of isolation during the scan did not, however, differ for patients although carers were more likely to report feeling cut off from their relative during the PET scan (mean (SD) 3.5 (1.2)) than SPECT (mean (SD) 4.2 (0.9), $p<0.001$, Wilcoxon signed ranks test). Carers were also 
more likely to report that their relative was anxious prior to the PET scan (mean (SD) 3.1 (1.2)) than SPECT (mean (SD) 3.3 (1.3), $p=0.03$, Wilcoxon signed ranks test). For controls, the only significant difference between the two scans related to whether they found the temperature comfortable $(73.7 \%$ for PET vs $94.7 \%$ for SPECT, $p=0.02$, Wilcoxon signed ranks test).

Some significant differences between patients and controls were observed, with controls generally feeling more positive about the scans than patients. In relation to both PET and SPECT scans controls felt more positive prior to the scan (mean (SD) PET: $3.9(0.3)$ for controls and $3.2(0.8)$ for patients, $p<0.001$; SPECT: $3.8(0.4)$ for controls and $3.3(0.9)$ for patients, $p=0.003$, Mann Whitney $U$ test); they rated the explanation provided by staff more highly (mean (SD) PET: 4.0 (0.2) for controls and 3.8 (0.5) for patients, p=0.04; SPECT: 4.0 (0.2) for controls and 3.4 (1.2) for patients, $p=0.04$, Mann Whitney $U$ test); and they gave a higher overall rating for the scan on a seven-point scale (mean (SD) PET: $5.5(0.6)$ for controls and $5.0(0.7)$ for patients, $p<0.001$; SPECT: 5.5 (0.5) for controls and $5.0(0.8)$ for patients, $p=0.003$, Mann Whitney $U$ test). In addition, controls rated the SPECT scan as more comfortable than patients (mean (SD) 3.6 (0.7) for controls and 3.0 (1.1) for patients, $p=0.02$, Mann Whitney U test).

Since patients, controls and carers made similar comments to the open questions; the results for all respondents have been combined. The chance to help with research and the interaction with staff were identified as the most positive aspects of both scans. The most negative aspect of their experience related to the scan itself (e.g. feeling cold or uncomfortable). While the majority of respondents did not suggest any improvements to the procedures $(79 \%$ for SPECT and $82 \%$ for PET), there were a number of potentially useful suggestions. Most of these related to improving comfort during the scan $(41 \%$ of suggestions relating to PET and $52 \%$ of those relating to SPECT). These included: increasing the width of the scan bed (or providing arm rests); providing a pillow for under the 
knees; and providing a blanket (or increasing the ambient temperature). Other suggestions were to reduce waiting time; provide more information on what to expect and to provide more feedback during the scan (e.g. how long was left).

\section{Prioritising task}

None of the attributes was consistently viewed positively or negatively by all respondents (Figure 2). While patients and carers valued the presence of a companion during the scan this was not important for controls ( $p<0.001$, Kruskal Wallis). Controls rated the noise of the scanner as more important and patients gave a lower rating to feeling confined in the scanner (both $p<0.05$, Kruskal Wallis) (Figure 2).

[Insert Figure 2 about here please]

\section{Willingness to pay}

Controls did not have a significant scan preference; carers had a marginal preference for SPECT over PET (one sample Wilcoxon signed ranks test, $p=0.05$ ). Nearly three quarters of all respondents with a preference were willing to pay for the preferred scan (independent of the scan preferred). Although the amounts respondents were willing to pay for SPECT (mean £1632, SD 1858, median £1000) were higher than for PET (mean £1074, SD 2197 , median £100) this was not significantly different (Mann Whitney $U$ test). In the hypothetical situation that the non-preferred scan proved more accurate, all respondents who had initially expressed a preference stated that they would prefer the more accurate scan. Of those without an initial preference, the majority (93\%) would prefer the more accurate scan.

\section{Physiological measures}

There were some technical difficulties in obtaining pulse rate data; the number of cases used at each data point for analysis ranged from 71 (48 patients and 23 controls) to 92 (66 patients and 26 controls). Since there were no significant differences in mean pulse rate 
between patients and controls at any data point; data from both groups were combined. While mean pulse rate did not change between the home visit (HV) and pre-SPECT data points, it decreased significantly (but numerically very slightly) when attending for a PET scan (from 67.9 (SD 11.3) to 65.6 (SD 11.5), p<0.05, analysis of variance, Figure 3). For both types of scan, pulse rate was significantly lower during the scan than on arrival at the clinic (both $p<0.001$, analysis of variance). Compared to beginning of the scan, pulse rate significantly lowered towards the end of the PET scan $(p<0.001$, analysis of variance); in contrast, while pulse rate declined gradually throughout the longer SPECT scan the difference did not reach statistical significance.

[Insert Figure 3 about here please]

\section{Discussion}

This study is the first to compare consumer views of PET and SPECT brain imaging for diagnosis of dementia. Our findings suggest that the two types of scans were equally acceptable to the majority of people with dementia, their carers and to healthy controls. There was also evidence that, even where a preference for one scan was expressed, diagnostic accuracy overruled any initial preferences suggesting that this was more important than other scan characteristics. Economic theory would use the willingness to pay amounts as representative of the strength of preference for each scan. The mean amounts respondents were willing to pay were not significantly different between PET and SPECT again confirming the lack of a strong preference for either scan. Pulse rate data indicated that for most participants, the experience of brain imaging was no more stressful than completing questionnaires in their own homes. Although some statistically significant differences were observed in heart rate, the effect size was very small and not of clinical significance. A previous study similarly found that SPECT and MRI imaging procedures had little impact on heart rate (Sparrow et al., 2004). 
It is of interest that participants' views and preferences seemed unaffected by the duration of the scan (SPECT scans took over twice as long as PET scans) or the physical surroundings. While SPECT scans took place in a busy hospital department, PET scans took place at a University Research Facility in a quiet clinic with typically only one patient attending at a time. Responses showed that some participants preferred the busier clinic since they enjoyed chatting with other patients whilst waiting for their scan. The importance of individual preferences was also evident on questions relating to comfort during the scan. Up to one fifth of participants felt cold during scans suggesting that either the ambient temperature needs to be increased or blankets need to be provided. Some participants would have welcomed pillows or eye masks. While blankets and pillows were available, these were not routinely offered to patients. The results highlight the need for a person-centred approach, addressing the needs of individual patients. Staff may also benefit from training in communicating with people with dementia to ensure that patients have a better understanding of the procedures they are to undergo.

A key difference between PET and SPECT scans was that carers were able to remain with the patient throughout SPECT scans, but not during the uptake and scanning phase of PET in order to keep radiation exposure as low as reasonably achievable (ALARA principle). Previous research has shown that a high proportion of older patients, particularly those with dementia, are accompanied to medical consultations (Ishikawa et al., 2006). Having a companion present can reassure both the patient and their carer and may enhance the quality and even feasibility of a successful scan. Over one third of patients and carers felt having a companion during the scan was important. Guidance allows for 'comforters and carers' to legally exceed the normal radiation dose limits, providing that they do so knowingly and willingly (i.e. doses have been estimated and explained to them)(Singleton et al., 2003). Available evidence indicates that the dose for companions waiting with a patient in the FDG uptake phase is surprisingly low (Singleton et al., 2003), suggesting scope for increased flexibility regarding the presence of carers throughout PET scans with vulnerable patient 
groups, such as people with dementia. Alternative approaches to allowing patients to remain in contact with their carer during PET scans, such as intercom systems, could also be considered.

\section{Implications for research}

The questionnaires about patient views and experiences of each scan were completed immediately after the scan to minimise problems with recall. While some patients with dementia were not able to recall all details of the scan, they successfully completed the majority of questions, confirming that this approach was acceptable and feasible for people with dementia. While only one study using Q methodology with people with Alzheimer's disease has been reported (Forrest, 2000) this approach has some similarities to Talking Mats which can facilitate communication with people with dementia (Murphy et al., 2010). The majority of people with dementia successfully completed our simplified version of $Q$ methodology confirming that this can be a useful approach for exploring the views and preferences of people with dementia.

\section{Strengths and limitations of the study}

The use of different approaches to data collection allowed us to explore preferences in detail and increases confidence in our findings. Although the questionnaires relating to each scan were identical, different members of staff administered the questionnaires after PET and SPECT and this could potentially have influenced the results. Participants in the study were not attending for routine scans but had volunteered to take part in a research study; their views towards the scans may potentially be more positive than those of patients attending for diagnostic purposes. The different settings for PET and SPECT scans in the present study could have influenced our results (although there was no evidence of a consistent preference for either setting). Future studies could explore patient views on PET and SPECT scans conducted in more similar environments. There were some problems with obtaining readings from the fingertip pulse oximeter. There is, however, no reason to suggest that the 
participants with missing data differed systematically from those with complete pulse rate readings. Although we did not adjust for multiple comparisons this was to ensure that we captured any differences between participant views of PET and SPECT and increases our confidence in concluding that, from the perspective of participants, there are minimal differences between the two imaging procedures.

\section{Conclusion}

The majority of participants viewed the experience of PET and SPECT scans as roughly equivalent although carers had a marginal preference for SPECT. Diagnostic accuracy was prioritised over other characteristics of scans (e.g. time taken, comfort on the scanner). The presence of a companion was important to some people with dementia and carers and may explain carers' preference for SPECT. Pulse rate data indicated that for most participants, the experience of brain imaging was no more stressful than completing standardised questionnaires in their own homes. Possible ideas for service development include a more person-centred approach to improve comfort during scans and a more flexible approach to the presence of carers during PET scans. Overall our findings indicate that the decision whether to use PET or SPECT scans in dementia should be based on diagnostic accuracy rather than other considerations. 


\section{Conflict of interest}

MF reports grants from National Institute for Health Research, JOB reports grants from GE Healthcare and Lilly; and other from GE Healthcare, Lilly, Bayer Healthcare, TauRx, and Cytox outside submitted work.

Word count 5204

\section{Description of authors' roles}

CB contributed to study conception, design, obtaining funding, data analysis, data interpretation and writing of the manuscript.

$\mathrm{KO}$ contributed to recruitment and data collection.

CDa contributed to recruitment, data collection and analysis.

NB contributed to recruitment, data collection and project management of the study.

$\mathrm{JL}$ contributed to study design and management.

DW contributed to study design, obtaining funding, organisation and management of the project.

MF contributed to study design, data analysis and data interpretation.

HM contributed to data analysis and data interpretation.

CDo contributed to study conception, design, obtaining funding and data interpretation. JO'B contributed to study conception, design, obtaining funding, organisation and management of the project, data analysis and data interpretation.

All authors contributed to the drafting of this paper

\section{Acknowledgements}

This paper summarises independent research funded by the National Institute for Health Research (NIHR) under its Research for Patient Benefit Programme (Grant reference number NIHR RfPB PB-PG 1207 13105). The study was also supported by the NIHR Biomedical Research Centre in Ageing and Chronic Disease and Biomedical Research Unit in Lewy-body Dementia (Newcastle upon Tyne Hospitals NHS Foundation Trust and 
Newcastle University) and the Biomedical Research Centre and Unit in Dementia (Cambridge University Hospitals NHS Foundation Trust and the University of Cambridge). We thank the Dementia and Neurodegenerative Diseases Research Network (DeNDRoN) for valuable support with recruitment. The views expressed are those of the authors and do not necessarily reflect those of the RfPB programme, NIHR, NHS or the Department of Health. 


\section{References}

Baker, R., Thompson, C. and Mannion, R. (2006). Q methodology in health economics. Journal of Health Services Research and Policy, 11, 38-45.

Colloby, S. and O'Brien, J. (2004). Functional imaging in Parkinson's disease and dementia with Lewy bodies. Journal of Geriatric Psychiatry \& Neurology, 17, 158-163.

Davison, C. M. and O'Brien, J. T. (2013). A comparison of FDG-PET and blood flow SPECT in the diagnosis of neurodegenerative dementias: a systematic review. International Journal of Geriatric Psychiatry, n/a-n/a.

Davison, C. M. and O'Brien, J. T. (2014). A comparison of FDG-PET and blood flow SPECT in the diagnosis of neurodegenerative dementias: a systematic review. International Journal of Geriatric Psychiatry, 29, 551-561.

Donaldson, C. and Shackley, P. (1997). Does "process utility" exist? A case study of willingness to pay for laparoscopic cholecystectomy. Social Science \& Medicine, 44, 699707.

Folstein, M. F., Folstein, S. E. and McHugh, P. R. (1975). Mini-mental state: a practical method for grading the cognitive state of patients for the clinician. Journal of Psychiatric Research, 12, 189-198.

Forrest, J. (2000). Using Q Methodology to Assess Chronic Pain in Elderly Cognitively Intact and Alzheimer's Patients. Operant Subjectivity, 24, 38-48.

Gauthier, S., et al. (2012). Recommendations of the 4th Canadian Consensus Conference on the Diagnosis and Treatment of Dementia (CCCDTD4). Canadian Geriatrics Journal, 15. Herholz, K. (2011). Perfusion SPECT and FDG-PET. International Psychogeriatrics, 23 Suppl 2, S25-31.

Hort, J., et al. (2010). EFNS guidelines for the diagnosis and management of Alzheimer's disease. European Journal of Neurology, 17, 1236-1248.

Ishii, K. and Minoshima, S. (2005). PET is better than perfusion SPECT for early diagnosis of Alzheimer's disease -- for. European Journal of Nuclear Medicine \& Molecular Imaging, $32,1463-1465$. 
Ishikawa, H., Roter, D. L., Yamazaki, Y., Hashimoto, H. and Yano, E. (2006). Patients' perceptions of visit companions' helpfulness during Japanese geriatric medical visits. Patient Education and Counseling, 61, 80-86.

Liang, W., et al. (2003). Acceptability of diagnostic tests for breast cancer. Breast Cancer Research \& Treatment, 79, 199-206.

McKeith, I., et al. (2005). Diagnosis and management of dementia with Lewy bodies: third report of the DLB Consortium. Neurology, 65, 1863-1872.

McKhann, G., Drachman, D., Folstein, M., Katzman, R., Price, D. and Stadlan, E. M. (1984). Clinical diagnosis of Alzheimer's disease: report of the NINCDS-ADRDA Work Group under the auspices of Department of Health and Human Services Task Force on Alzheimer's Disease. Neurology, 34, 939-944.

Murphy, J., Gray, C. M., van Achterberg, T., Wyke, S. and Cox, S. (2010). The effectiveness of the Talking Mats framework in helping people with dementia to express their views on well-being. Dementia, 9, 454-472.

NICE/SCIE (2006). Dementia: supporting people with dementia and their carers in health and social care. London: National Institute for Clinical Excellence and Social Care Institute for Excellence.

O’Brien, J. T., et al. (2014). 18F-FDG PET and Perfusion SPECT in the Diagnosis of Alzheimer and Lewy Body Dementias. Journal of Nuclear Medicine, 55, 1959-1965.

Singleton, M., Griffiths, C., Morrison, G. and Soanes, T. (2003). Dose constraints for comforters and carers. Health and Safety Executive.

Sparrow, P., Plein, S., Jones, T. R., Thorley, P. J., Hale, C. and Sivananthan, M. U. (2004). Tolerance of MRI vs. SPECT myocardial perfusion studies--a patient survey. Journal of Magnetic Resonance Imaging, 19, 410-416.

Wollman, D. E., Beeri, M. S., Weinberger, M., Cheng, H., Silverman, J. M. and Prohovnik, I. (2004). Tolerance of MRI procedures by the oldest old. Magnetic Resonance Imaging, 22, 1299-1304. 
Table 1 Overview of study procedures

\begin{tabular}{lll} 
DATA TYPE & PARTICIPANTS & WHEN COLLECTED \\
\hline Questionnaire & Patients, controls \& carers & Immediately after each scan \\
Prioritising task & Patients, controls \& carers & Final home visit \\
Willingness to pay & Controls \& carers & Final home visit \\
Pulse rate & Patients \& controls & 5 minutes at initial home visit \\
& & 5 minutes on arrival for each scan \\
& & Throughout each scan \\
\hline
\end{tabular}


Figure 1 Patient \& control views on PET and SPECT

How are you feeling about having the scan today?

How satisfied were you with the length of time you had to wait between your appointment time and the injection?

How satisfied were you with the length of time you had to wait between the injection and the scan?

How comfortable were you with the distance between you and the scanner?

How quickly did the time pass while you were in the scanner?

How physically comfortable were you when lying on the scanner?

How acceptable was the noise of the scanner?

How isolated or cut-off from people did you feel during the scan? *

Did staff put you at your ease?

Did staff explain what was going to happen?

Given the time and effort involved in coming for the scan today, how worthwhile is this type of scan if it makes

a correct diagnosis for eight out of ten patients?

How would you rate the scan overall?

* - Scoring of item reversed
$0 \% \quad 20 \% \quad 40 \% \quad 60 \% \quad 80 \% \quad 100 \%$

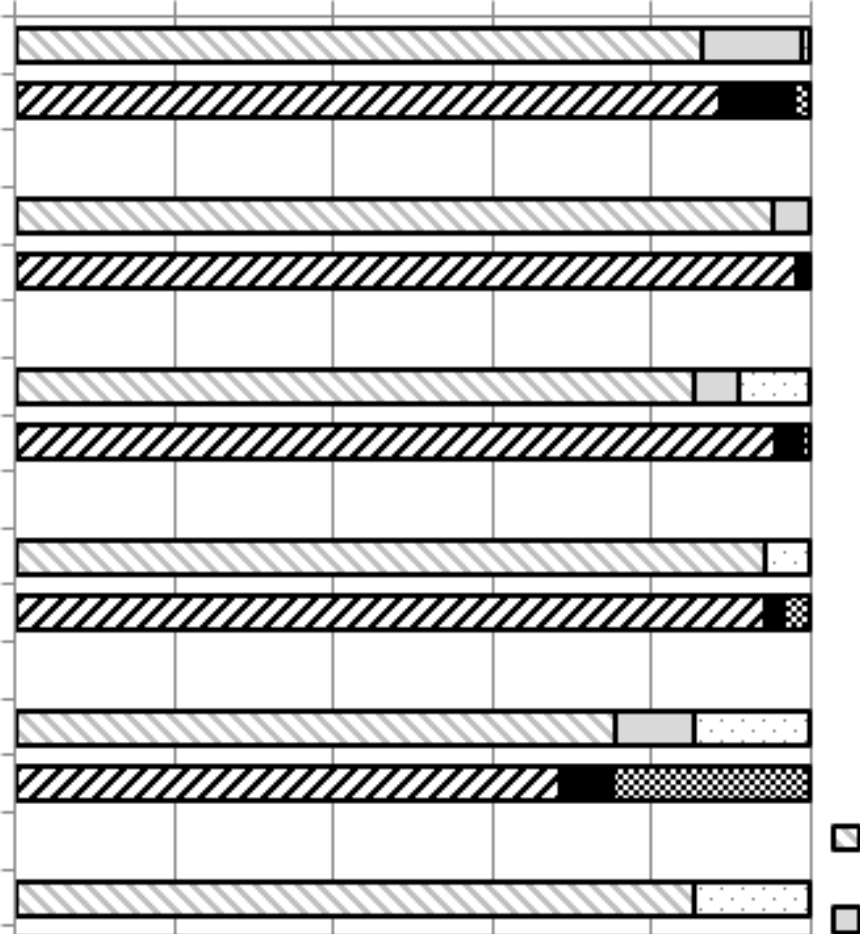

PET +
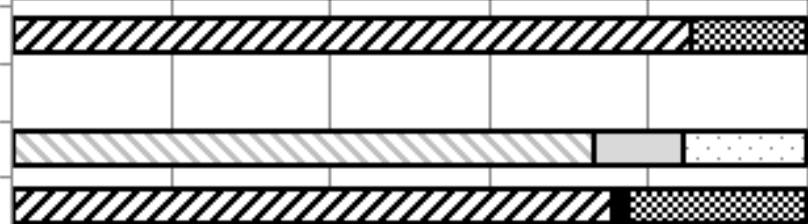

ØSPECT +

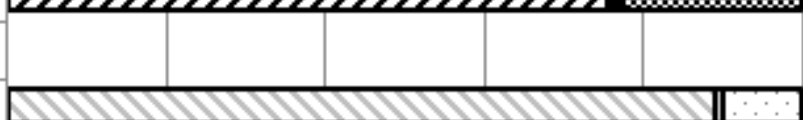

SPECT Neutral \$ SPECT -

PET Neutral

DPET -
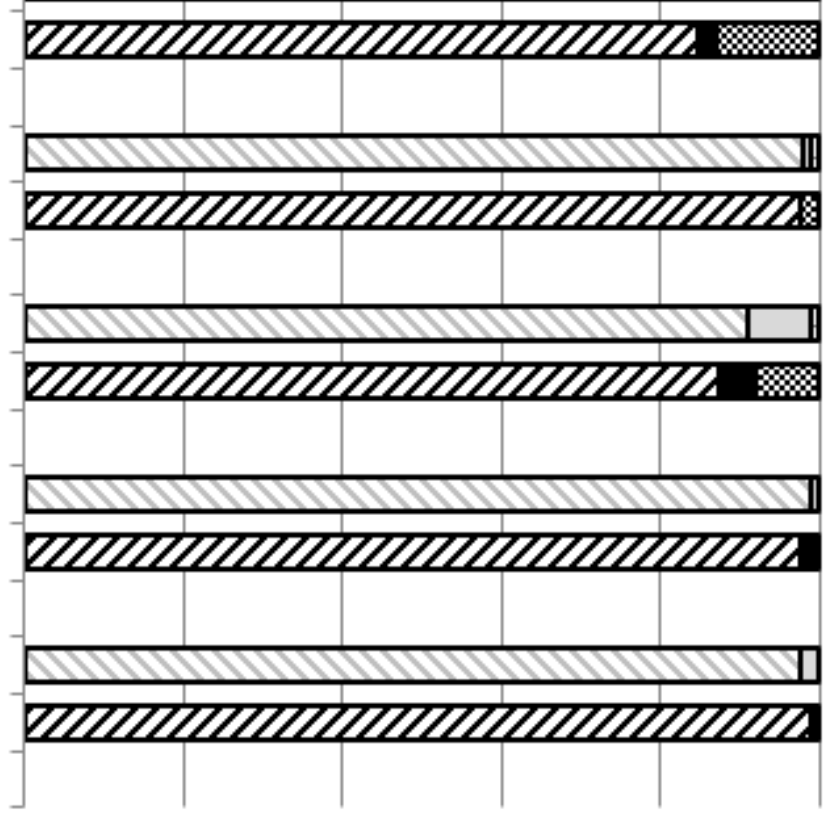
Figure 2 Percentage of respondents rating scan attributes as important

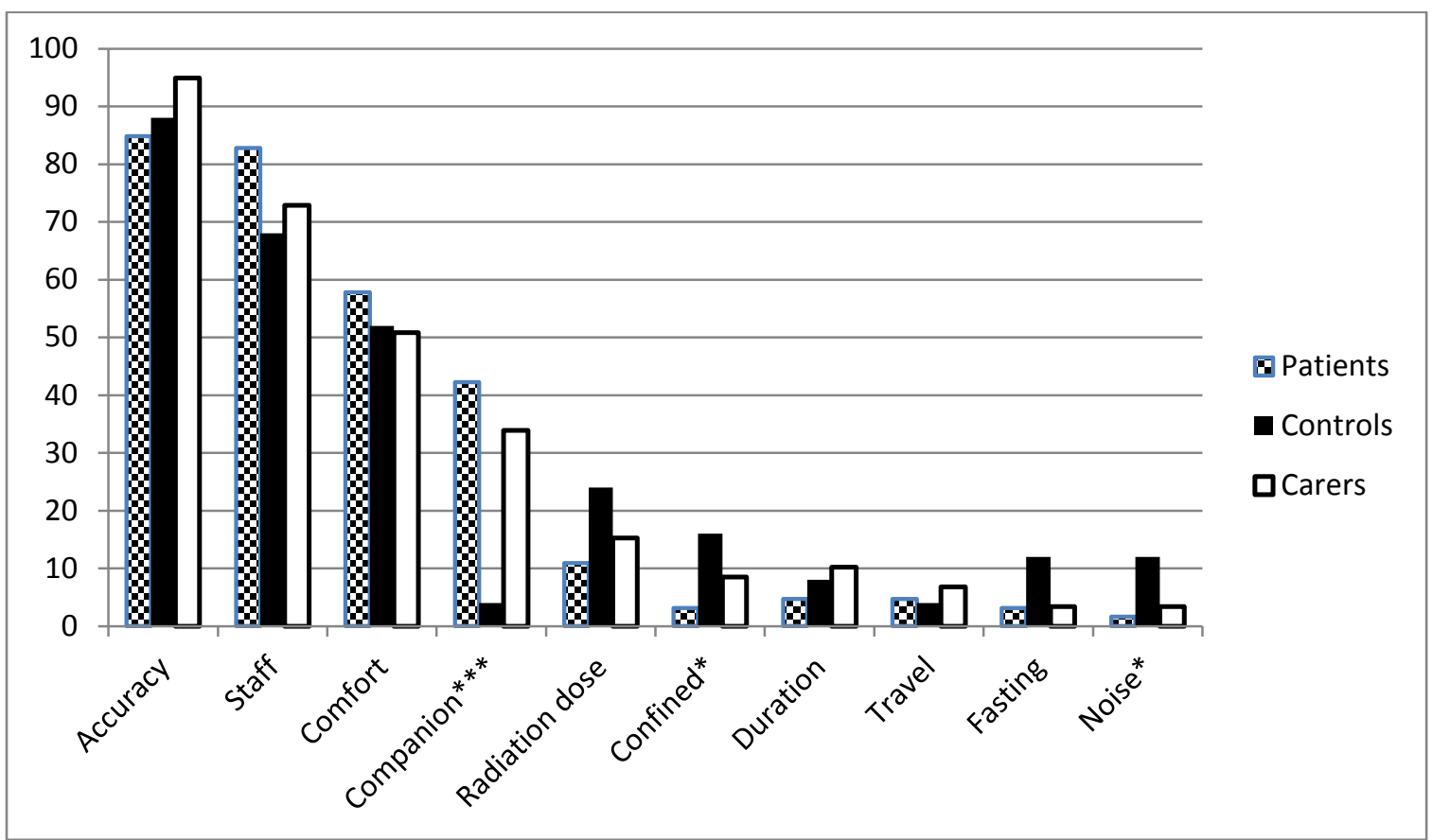

${ }^{*} p<.05 \quad{ }^{* * *} p<.001$ 
Figure 3 Mean heart rate readings at different time points for PET and SPECT scans

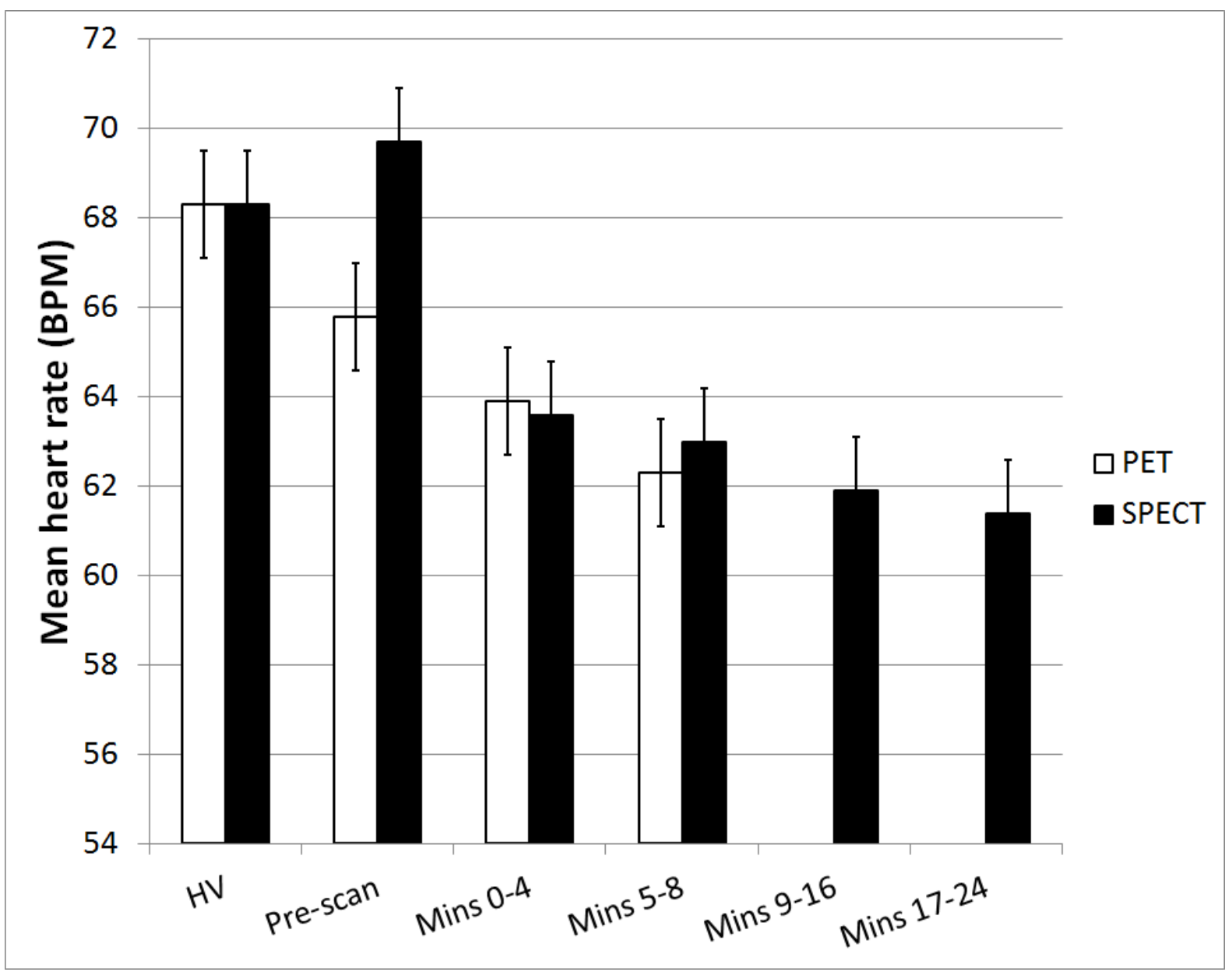

PET scans were completed within ten minutes therefore there are no data for later timepoints. 